\title{
Salud Oral en Población Escolar Urbana y Rural
}

\author{
Oral Health in Urban and Rural School Population
}

\author{
Consuelo Cabrera*; María Ignacia Arancet; Danitza Martínez*; Alfredo Cueto** \& Sebastian Espinoza***
}

CABRERA, C.; ARANCET, M. I.; MARTínEZ, D.; CUETO, A. \& ESPINOZA, S. Salud oral en población escolar urbana y rural. Int. J. Odontostomat., 9(3):341-348, 2015.

RESUMEN: La salud oral, está condicionada tanto por factores locales individuales como por factores socioeconómicos, culturales, ambientales, comunitarios y geográficos, entre estos últimos la condición urbano-rural cumple un rol importante. En un esfuerzo de vigilancia epidemiológica; se realizó el primer estudio en la zona que se fija como propósito comparar la salud oral en niños de 6 a 12 años de zonas rurales y urbanas, mediante indicadores de caries, estado de la salud periodontal y presencia de anomalías dentomaxilares. Estudio de prevalencia en escolares entre 6 y 12 años de zonas urbana y rural de la Región de Valparaíso. La muestra correspondió a 231 niños que presentaron dentición mixta o permanente. Las variables recogidas fueron edad, sexo, localidad, presencia de alteraciones mucosas, dentomaxilares y periodontales; índices ceod, COPD, índice de higiene oral; presencia de sellantes y necesidad de tratamiento; nivel educacional de jefe de hogar, del encargado del niño y situación laboral del jefe de hogar. La base de datos se analizó mediante Microsoft Excel y Stata 13.0. Se realizó estadística descriptiva e inferencial bivariante y multivariante. La presencia de caries, gingivitis generalizada y alteraciones mucosas fueron significativamente mayores en sector rural, al igual que la necesidad de tratamiento de anomalías dentomaxilares, restauraciones y caries. Los promedios de ceod y COPD fueron mayores en sector rural que urbano, con diferencias estadísticamente significativas. A través de un modelo lineal se observó que variables como educación del jefe de hogar y encargado del niño y situación laboral del jefe de hogar no resultaron significativas al intentar explicar la variabilidad de COPD y ceod, pero sí la localidad. La salud oral presentó mayor daño en niños de sector rural constituyéndose en factor de riesgo para caries, daño periodontal, anomalías dentomaxilares, y menor acceso a los servicios de salud.

PALABRAS CLAVE: salud oral, salud urbana, salud rural, caries dental.

\section{INTRODUCCIÓN}

En Chile al igual que a nivel mundial, las patologías orales son altamente frecuentes en especial caries, enfermedades gingivales/periodontales, anomalías dentomaxilares (OMS, 1997) y afectan en mayor proporción a la población vulnerable.

La salud oral, está condicionada tanto por factores locales individuales como por factores sociales, comunitarios, económicos, culturales, ambientales y geográficos, entre estos últimos la condición urbanorural, así ruralidad generalmente se ve ligada a un mayor nivel de pobreza, menor nivel educacional y menor nivel socioeconómico. En Chile según el XVII censo de población realizado del año 2002, un $13,4 \%$ de la población vive en áreas rurales (INE, 2003).
En los estudios poblacionales de salud oral efectuados en Chile en 2007, la probabilidad que los niños de 6 y 12 años estén libres de caries es 2 veces mayor en las zonas urbanas, en comparación con las áreas rurales, mientras que el daño por caries es mayor en las zonas rurales que en las urbanas (Ministerio de Salud, 2007).

Nuestro estudio constituye un esfuerzo de vigilancia epidemiológica para evaluar el estado de salud oral del sector rural y es el primero que entregará evidencia del estado de las principales enfermedades que afectan el territorio oral y máxilofacial en la población infantil según su distribución urbano rural en la región.

\footnotetext{
* Cirujano dentista, Facultad de Odontología, Universidad Andrés Bello, Viña del Mar, Chile.

* Cirujano dentista, Magister en Salud Pública, Profesor Titular, Facultad de Odontología, Universidad Andrés Bello, Viña del Mar, Chile.

"Kinesiólogo y Estadístico. Profesor Ayudante Cátedra de Salud Pública, Facultad de Odontología, Universidad Valparaíso, Valparaíso, Chile.
} 
El propósito del estudio es comparar la salud oral en niños de 6 a 12 años de zonas rurales y urbanas de la $V$ región, a través de indicadores de caries, de estado de la salud periodontal y presencia de anomalías dentomaxilares.

\section{MATERIAL Y MÉTODO}

Estudio de prevalencia, la población correspondió a escolares entre 6 y 12 años pertenecientes a establecimientos de educación básica de zonas urbana y rural de la Región de Valparaíso. El tamaño muestral se determinó utilizando metodología de muestreo para poblaciones infinitas, con un total de 231 niños. Se estimó la potencia de la prueba, una vez realizado el estudio, a través de la prueba de satterthwaite, asumiendo varianzas diferentes, entregando ésta una potencia del $91 \%$.

La selección se hizo a través de un muestreo por conglomerado, eligiendo dos comunas una urbana Viña del Mar y una rural Putaendo. Se fijó nivel de ruralidad según el índice de ruralidad entregados por INE en el año 2000. Selección probabilística de los establecimientos educacionales tanto en sector urbano como rural. Se incluyó a niños de los establecimientos seleccionados, con dentición mixta o permanente, que accedieron voluntariamente al examen clínico, previa autorización de sus padres. Se excluyó a los niños con mal comportamiento al momento del examen clínico y niños con discapacidad mental.

El examen clínico fue realizado por dos examinadoras, previamente calibrados obteniendo un kappa $=0,8$ (Buena concordancia) y se realizó de acuerdo a las recomendaciones de la OMS, apoyado en la utilización de linternas LED frontales (Eveready $\left.{ }^{\circledR}\right)$. Se tomaron las medidas pertinentes de bioseguridad, recomendadas por el MINSAL. El tiempo promedio de examen fue de 10 minutos por niño. Entre las variables sociodemográficas se recogieron edad, sexo del niño y localidad. Y entre las variables clínicas recogidas: Presencia de alteraciones mucosas, dentomaxilares (ADM) y periodontales (Gingivitis generalizada); índices clínicos: ceod, COPD, de higiene oral; presencia de sellantes y necesidad de tratamiento ambas (Presencia de restauraciones en mal estado, caries, ADM y gingivitis) en escala dicotómica.

Se aplicó encuesta a los apoderados de los niños para recoger variables socio demográficas y conductuales en salud oral: identificación parenteral del jefe de hogar, nivel educacional de jefe de hogar $y$ del cuidador o encargado del niño (Escala de medición: profesión universitaria, profesión técnica profesional, educación media completa, educación básica completa, educación básica incompleta, analfabeto), situación laboral de jefe de hogar.

Se solicitó la autorización del Encargado de departamento de educación de cada municipalidad y del director de cada establecimiento educacional. Los padres de los niños participantes debieron firmar un consentimiento informado y cada niño firmó un asentimiento informado. Un informe escrito con los resultados del examen clínico de cada niño fue entregado en forma confidencial a cada apoderado. Este estudio fue aprobado por el Comité de Investigación de la Facultad de Odontología Universidad Andrés Bello, Viña del Mar, Chile.

Para el análisis estadístico de la base de datos se utilizaron los software Microsoft Excel y Stata 13.0. Para las variables cualitativas, se utilizaron tablas de frecuencia y para las variables cuantitativas se calcularon medidas descriptivas: promedio, mediana, desviación estándar con sus respectivos intervalos de confianza al 95\%. Para el análisis inferencial se utilizaron los test estadísticos: Pruebas de normalidad de Shapiro-Wilk, prueba $Z$ para proporciones, prueba $T$ para medias con varianza desconocida, ANOVA de 1 y 2 factores, regresión lineal simple y en el caso de variables no paramétricas, pruebas de Mood y Kruskal Wallis.

\section{RESULTADOS}

Este estudio recogió datos de 231 niños. El $29,4 \%$ del total correspondieron a niños de sector rural $(n=68)$ y $70,56 \%$ de sector urbano $(n=163)$. El promedio de edad fueron $8,26 \pm 1,5$ años y en relación al sexo, $45 \%$ eran hombres y $55 \%$ mujeres.

En relación al porcentaje de niños afectados por caries, gingivitis generalizada y alteraciones mucosas por localidad, se obtuvieron diferencias estadísticamente significativamente, pues el daño fue mayor en el sector rural, como se observa en la Tabla I.

Respecto a anomalías dentomaxilares según tipo, detallada en la Tabla II, se presentó mayor daño en el sector rural, con diferencias estadísticamente sig- 
CABRERA, C.; ARANCET, M. I.; MARTíNEZ, D.; CUETO, A. \& ESPINOZA, S. Salud oral en población escolar urbana y rural. Int. J. Odontostomat., 9(3):341-348, 2015.

Tabla I. Distribución de principales patologías orales según sector de residencia.

\begin{tabular}{|c|c|c|c|c|c|c|}
\hline \multirow{2}{*}{$\begin{array}{l}\text { Variable en estudio } \\
\text { Presencia Caries }\end{array}$} & \multirow{2}{*}{$\begin{array}{l}\text { Sector } \\
\text { Rural }\end{array}$} & \multirow{2}{*}{$\begin{array}{c}\text { Proporción afectados } \\
88,23 \%\end{array}$} & \multirow{2}{*}{$\begin{array}{c}\text { ES } \\
0,004\end{array}$} & \multicolumn{2}{|c|}{ Inter. Confianza 95\% } & \multirow{3}{*}{$\begin{array}{l}\text { Valor F } \\
0,000\end{array}$} \\
\hline & & & & $80,57 \%$ & $95,89 \%$ & \\
\hline & Urbano & $53,37 \%$ & 0,004 & $45,71 \%$ & $61,03 \%$ & \\
\hline \multirow{2}{*}{ Gingivitis Generalizadas } & Rural & $81,25 \%$ & 0,005 & $70,20 \%$ & $92,29 \%$ & \multirow[b]{2}{*}{0,000} \\
\hline & Urbano & $40,81 \%$ & 0,004 & $31,08 \%$ & $50,54 \%$ & \\
\hline \multirow{2}{*}{ Alteraciones } & Rural & $19,11 \%$ & 0,004 & $9,77 \%$ & $28,46 \%$ & \multirow{2}{*}{0,000} \\
\hline & Urbano & $7,54 \%$ & 0,002 & $3,44 \%$ & $11,65 \%$ & \\
\hline
\end{tabular}

Tabla II. Distribución de tipos de anomalías dentomaxilares según sector de residencia.

\begin{tabular}{llccccc}
\hline Variable en estudio & Sector & Proporción afectados & ES & Int. confianza 95\% & Valor P \\
\hline \multirow{2}{*}{ Compresión maxilar } & Rural & $18,18 \%$ & 0,04 & $8,87 \%$ & $27,48 \%$ & \multirow{2}{*}{0,001} \\
& Urbano & $5,03 \%$ & 0,01 & $1,63 \%$ & $8,42 \%$ & \\
\multirow{2}{*}{ Mordida cruzada } & Rural & $29,41 \%$ & 0,05 & $18,58 \%$ & $40,24 \%$ & 0,051 \\
& Urbano & $19,49 \%$ & 0,03 & $13,33 \%$ & $25,65 \%$ & \\
\multirow{2}{*}{ Mordida vis a vis } & Rural & $13,23 \%$ & 0,04 & $5,18 \%$ & $21,28 \%$ & 0,119 \\
& Urbano & $8,17 \%$ & 0,02 & $3,91 \%$ & $12,43 \%$ & \\
\multirow{2}{*}{ Sobremida abierta } & Rural & $23,52 \%$ & 0,05 & $13,44 \%$ & $33,61 \%$ & 0,000 \\
& Urbano & $4,40 \%$ & 0,01 & $1,21 \%$ & $7,59 \%$ & \\
\multirow{2}{*}{ Distoclusión } & Rural & $14,70 \%$ & 0,04 & $6,28 \%$ & $23,12 \%$ & 0,481 \\
\multirow{2}{*}{ Mesioclu sión } & Urbano & $14,46 \%$ & 0,02 & $8,99 \%$ & $19,93 \%$ & \\
& Rural & $50,0 \%$ & 0,06 & $38,11 \%$ & $61,88 \%$ & 0,414 \\
& Urbano & $48,42 \%$ & 0,03 & $40,65 \%$ & $56,19 \%$ & \\
& Rural & $25,0 \%$ & 0,05 & $14,70 \%$ & $35,29 \%$ & 0,007 \\
\hline
\end{tabular}

nificativas respecto al sector urbano en compresión maxilar, mordida abierta y mesioclusión.

Al comparar la necesidad de tratamiento según localidad, se obtuvieron diferencias estadísticamente significativamente, para anomalías dentomaxilares, restauraciones y caries siendo mayores en sector rural, como se observa en la Tabla III.

En relación al índice de higiene oral, se observó que un $88,2 \%$ de los niños de zona rural presentaron un índice de higiene oral entre bajo y medio (IC $80,6 \%-95,9 \%$ ) mientras que el $80,5 \%$ de los niños de residencia urbana presentaron un índice de higiene oral entre bajo y medio (IC $74,3 \%-86,7 \%)$, sin diferencias estadísticamente significativas (valor $p$ 0,07) entre grupos.

Respecto a los dientes sellados por localidad, se obtuvo que el promedio para dientes sellados en rural fue 2,6 mientras que para urbano fue 4,1 , con diferencias estadísticamente significativas (valor $p 0,0005$ ).

Tanto al comparar COPD y ceod por localidad, se obtuvo que el promedio de COPD para rural fue $1,01$ (IC $0,74-1,44)$, mientras que para urbano se obtuvo un promedio de 0,52 (IC $0,37-0,68$ ) y el promedio de ceod para rural fue 3,66 (IC 2,99-4,33) mien-

Tabla III. Necesidad de tratamiento según localidad.

\begin{tabular}{|c|c|c|c|c|c|c|}
\hline \multirow{3}{*}{$\begin{array}{l}\text { Necesidades de tratamiento } \\
\text { Glngivitis }\end{array}$} & \multirow{3}{*}{$\begin{array}{c}\text { Sector } \\
\text { Rural } \\
\text { Urbano }\end{array}$} & \multirow{3}{*}{$\begin{array}{c}\text { Proporción afectados } \\
69,12 \% \\
62,26 \%\end{array}$} & \multirow{3}{*}{$\begin{array}{c}\text { ES } \\
0,06 \\
0,04\end{array}$} & \multicolumn{2}{|c|}{ Int. Confianza 95\% } & \multirow{3}{*}{$\begin{array}{l}\text { Valor } \mathbf{P} \\
0,162\end{array}$} \\
\hline & & & & $58,14 \%$ & $80,10 \%$ & \\
\hline & & & & $54,73 \%$ & $69,80 \%$ & \\
\hline \multirow{2}{*}{ Anomalías Dentomaxilares } & Rural & $73,53 \%$ & 0,05 & $63,04 \%$ & $84,02 \%$ & \multirow{2}{*}{0,000} \\
\hline & Urbano & $44,03 \%$ & 0,04 & $36,31 \%$ & $51,74 \%$ & \\
\hline \multirow{2}{*}{ Restauraciones } & Rural & $55,88 \%$ & 0,06 & $44,08 \%$ & $67,68 \%$ & \multirow{2}{*}{0,023} \\
\hline & Urbano & $41,51 \%$ & 0,04 & $33,85 \%$ & $49,17 \%$ & \\
\hline \multirow[b]{2}{*}{ Caries } & Rural & $88,24 \%$ & 0,04 & $80,58 \%$ & $95,90 \%$ & \multirow[b]{2}{*}{0,000} \\
\hline & Urbano & $50,94 \%$ & 0,04 & $43,17 \%$ & $58,71 \%$ & \\
\hline
\end{tabular}


tras que en urbano fue 2,06 (IC 1,65-2,47) mostrando tanto en COPD y ceod diferencias estadísticamente significativas por localidad, valor p 0,0004 y 0,000 , respectivamente.

La distribución de los índices ceod y COPD por localidad se observa en la Figura 1 constatándose que existe mayor variabilidad de los datos en sector rural, tanto para COPD como ceod.

Al comparar índices COPD y ceod por edad y localidad, los resultados mostraron que los índices fueron siempre mayores en el sector rural como se constata en Tabla IV.
A través de modelos lineales para COPD, resulto que la única variable explicativa fue localidad, las restantes variables en los diferentes modelos de la tabla $V$ no resultan significativas, con valores $\mathrm{R} 2$ bajos. No obstante si aislamos la variable localidad y si aplicamos la prueba no paramétricas de Kruskal-Wallis al resto de variables, arroja resultados que indican diferencias en los valores medianos de COPD según las variables estudiadas. Ahora, analizando la magnitud de posibles asociaciones mediante la prueba de Spearman se puede ver que existen relaciones inversamente proporcionales pero que no se ven reflejadas en las pruebas de independencia con p-valores mayores a 0,05 para estas variables.

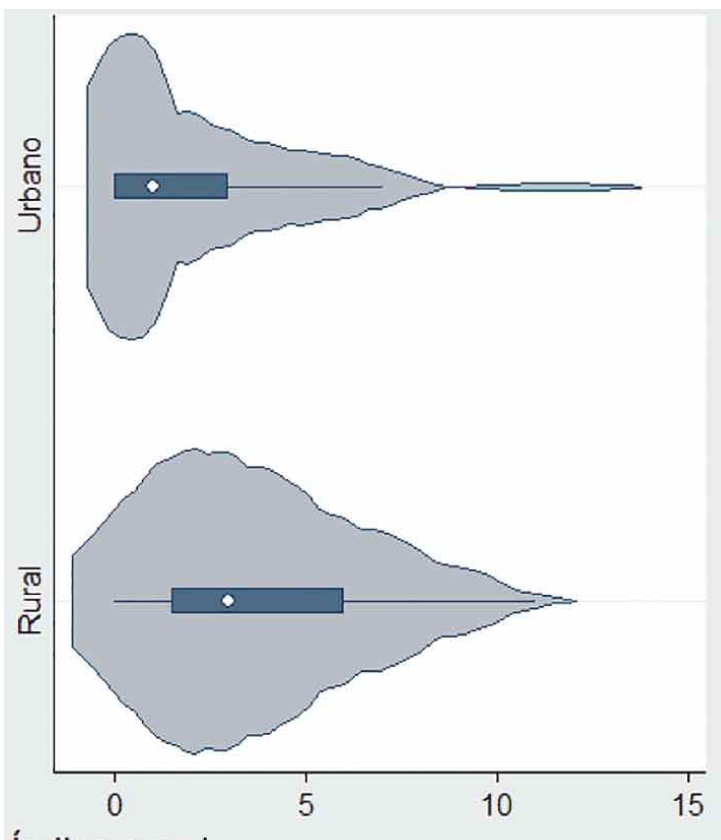

Índice ceod

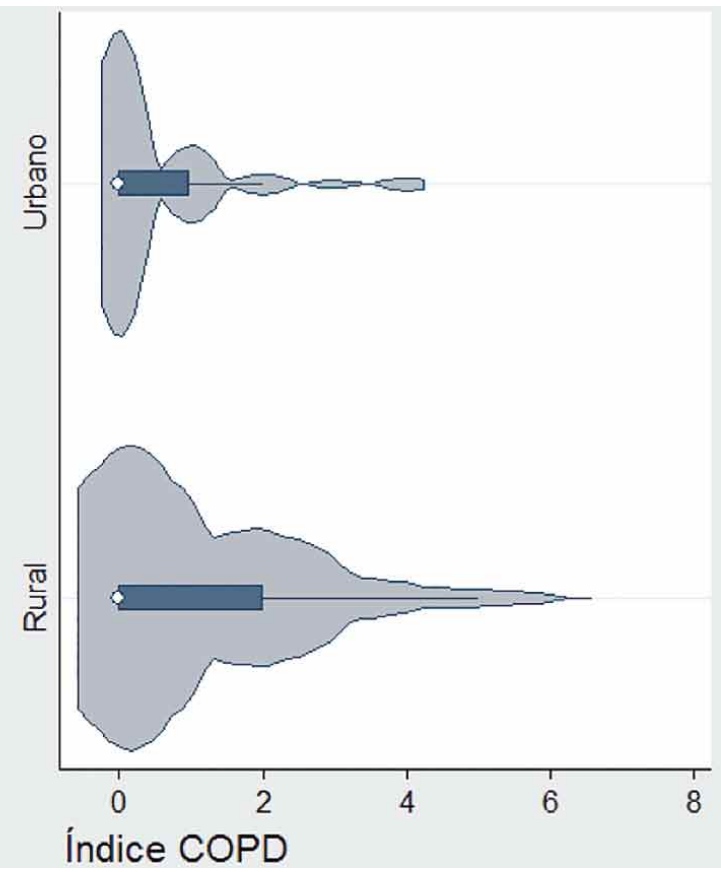

Fig. 1. Distribución de índice ceod, COPD según localidad.

Tabla IV. Comparación de índice ceod, COPD por años de edad y localidad.

\begin{tabular}{lcccccccc}
\hline & \multicolumn{3}{c}{ Edad } & \multicolumn{3}{c}{ Rural } & \multicolumn{3}{c}{ Localidad } & \multicolumn{3}{c}{ Urbano } & \\
\cline { 2 - 9 } & $\mathbf{n}$ & $\%$ & ceod & COPD & $\mathbf{n}$ & $\%$ & ceod & COPD \\
\hline 6 & 10 & 4,33 & 5,80 & 0,50 & 22 & 9,52 & 3,68 & 0,09 \\
7 & 10 & 4,33 & 4,70 & 1,10 & 40 & 15,32 & 2,17 & 0,55 \\
8 & 19 & 8,23 & 3,05 & 0,68 & 28 & 12,12 & 2,61 & 0,65 \\
9 & 22 & 9,52 & 3,09 & 1,23 & 32 & 13,85 & 1,63 & 0,57 \\
10 & 3 & 1,3 & 4,33 & 2,00 & 25 & 10,82 & 1,24 & 0,44 \\
11 & 3 & 1,3 & 1,67 & 2,33 & 12 & 5,19 & 0,92 & 1,00 \\
12 & 1 & 0,43 & 0,00 & 5,00 & 4 & 1,73 & 0,00 & 0,50 \\
Total & 68 & 29,44 & 3,66 & 1,09 & 163 & 70,56 & 2,06 & 0,52 \\
\hline
\end{tabular}


Tabla V. Variables independientes y su relación con variabilidad de COPD.

\begin{tabular}{|c|c|c|c|c|c|c|c|c|}
\hline \multirow[b]{2}{*}{ Modelo } & \multicolumn{2}{|c|}{ Modelo Lineal } & \multicolumn{6}{|c|}{ COPD } \\
\hline & \multicolumn{2}{|c|}{ Variables explicativa } & \multirow{3}{*}{$\begin{array}{c}\text { Coef, } \mathbf{R}^{2} \\
5,34 \%\end{array}$} & \multirow{3}{*}{$\begin{array}{c}\text { Kruskal-wallis } \\
\text { Valor } \mathbf{P} \\
0,004\end{array}$} & \multicolumn{2}{|c|}{$\begin{array}{c}\text { Spearman } \\
\text { Valor } \mathbf{P}\end{array}$} & \multicolumn{2}{|c|}{$\begin{array}{c}\text { Independiancia } \\
\text { Valor P }\end{array}$} \\
\hline \multirow[b]{2}{*}{1} & Localidad & Nivel educacional & & & Rural & $-0,13$ & Rural & 0,29 \\
\hline & 0,006 & $\begin{array}{c}\text { Jefe de hogar } \\
0,259\end{array}$ & & & Urbano & $-0,11$ & Urbano & 0,15 \\
\hline \multirow[b]{2}{*}{2} & Localidad & Nivel educacional & & & Rural & $-0,18$ & Rural & 0,13 \\
\hline & 0,001 & $\begin{array}{c}\text { encargado del niño } \\
0,097\end{array}$ & $5,92 \%$ & 0,04 & Urbano & $-0,10$ & Urbano & 0,21 \\
\hline \multirow{2}{*}{3} & Localidad & Situación laboral del & & & Rural & $-0,21$ & Rural & 0,08 \\
\hline & 0,001 & $\begin{array}{c}\text { Jele de Hogar } \\
0,42\end{array}$ & $5,45 \%$ & 0,092 & Urbano & $-0,07$ & Urbano & 0,34 \\
\hline
\end{tabular}

\section{DISCUSIÓN}

En nuestro estudio el daño de la salud oral fue consistentemente mayor en niños del sector rural que el urbano, coincidente con otros estudios en Chile y de otros países independientes del tramo de edad (Soto et al., 2007; Zander et al., 2013; Skinner et al., 2006; Geus et al., 2013). Tanto caries, gingivitis, anomalías dentomaxilares y alteraciones de la mucosa oral fueron significativamente más prevalentes en los niños de sectores rurales, es probable que esto se relacione con un problema de pobreza y vulnerabilidad.

Nuestro estudio abarcó un grupo etario de niños entre 6 a 12 años, con un promedio de edad de 8,26 (DE 1,5) años, lo que dificulta su comparabilidad con estudios nacionales, pero para estos efectos usaremos de referencia el estudio realizado por Soto et al., a los 12 años.

Geus et al. en niños con promedio de edad comparable al de nuestro estudio (8,1 años para sector rural y 7,9 años para sector urbano) informa de porcentajes de niños libres de caries para sector rural de $13,3 \%$ y urbano $36,9 \%$, obteniendo medias de COPD para sector rural 1,0 y urbano 0,39 , y medias de ceod para sector urbano 2,19 y rural 3,87 (Geus et al.), todos resultados similares a nuestro estudio lo que se podrían explicar por semejante nivel de desarrollo socioeconómico del sector rural tanto en Chile como Brasil. Por otro lado en Italia en niños de 8 años, el porcentaje libres de caries fue $44,1 \%$ en sector rural (Perinetti et al., 2006) es decir 4 veces más daño en sector rural que en nuestro estudio, lo que demuestra que se pueden implementar políticas públicas que mejoren sustantivamente la condición de salud oral de los niños en el sector rural en Chile.
En nuestro estudio el sector urbano fue representado por Viña del Mar cuidad con mas de 350.000 habitantes, obteniendo un ceod de 2,06 $(1,652,47)$ incluso menor al compararlo con la media nacional 3,71 $(3,56-3,86)$ dientes informada por Soto et al., (21) estos resultados se podrían explicar por amplias coberturas en salud oral, nivel socioeconómico y que la cuidad cuenta con agua potable fluorada hace más de tres décadas.

En relación al comportamiento de los índices de daño por caries, se observó una disminución constante de índice ceod al aumentar la edad de los niños, esto se explicaría por la pérdida de dientes temporales obturados y/o cariados con los años y por la exfoliación producto de la erupción de los definitivos. En el caso del COPD se observó que a mayor edad, el índice aumentó.

En los tres modelos de regresión realizados en este estudio, la localidad explica de forma significativa la variabilidad de COPD y ceod, sin embargo hay otras variables que podrían explicar estos indicadores como educación de jefe de hogar, educación del encargado del niño y situación laboral del jefe de hogar, sin embargo estas variables en nuestros modelos no fueron estadísticamente significativas y se necesitaría una muestra de mayor tamaño para determinar su impacto en la variabilidad de estos índices. Otros estudios mostraron que variables tales como nivel socioeconómico, hábitos de higiene oral, hábitos alimenticios, uso de fluoruros tópicos y sistémicos influirían en la variabilidad de COPD (Ministerio de Salud; Maupome et al., 2013). 
Los niños de sector rural contaban con el Programa de Alimentación Escolar con Leche Fluorada, lo cual constituye una medida preventiva para la reducción de los índices de caries, pues existe evidencia tanto a nivel local como internacional, en relación a la efectividad de la leche como vehículo de fluoruros de acción sistémica. En estudios clínicos realizados en diversos países la reducción de caries osciló entre $31,2 \%$ y $70 \%$ para dentición permanente y $14,8 \%$ y $40,1 \%$ para dentición temporal (Mariño et al., 2006). A nivel nacional, un estudio realizado en la localidad de Codegua que midió la reducción de caries en dientes temporales luego de 4 años de exposición a leche fluorada, obtuvo una reducción entre $40 \%$ a $53 \%$ para ceod y de $41 \%$ a $78 \%$ para ceos (Mariño et al., 1999). Si bien es cierto los niños de sector rural en nuestra investigación estaban cubiertos por el programa de leche fluorada, los índices de caries fueron mayores que en sector rural. Esto podría explicarse, entre otras cosas, porque los niños reciben la leche fluorada sólo en los establecimientos educacionales y su asistencia a estos no es constante, por lo cual con la aplicación de programas comunitarios complementarios de fluoración tópica mediante el uso de geles y barnices con alto contenido de fluoruros, podría contribuir a disminuir los altos índices de caries, pues es sabido que el uso de fluoruros sistémicos no reemplaza al uso de tópico (Mariño et al., 2006).

Por otro lado, la prevalencia de alteraciones periodontales, medida por la presencia de gingivitis localizada, arrojó una prevalencia de $40,81 \%$ en sector urbano y $81,25 \%$ en sector rural. Los datos nacionales obtenidos por Soto et al., también reportaron una prevalencia de gingivitis mayor para el sector rural que urbano, con $78,9 \%$ y $64,9 \%$, respectivamente. Si bien es cierto hay diferencias estadísticamente significativas en el daño periodontal entre sector urbano rural, la salud periodontal constituye un desafío relevante para ambos sectores que debiera resolverse por medio estrategias que entre otras cosas considere el control periódico e intervenciones preventivas promocionales a nivel de establecimientos educacionales.

El acceso a la salud se registró indirectamente a través de cantidad de sellantes en boca entregando diferencias significativas entre urbano y rural, con una media de sellantes de 4,1 y 2,6 , respectivamente, es reconocido que la utilización de sellantes de fosas y fisuras es una de las medidas más efectivas para la prevención de lesiones cariosas (Faleiros Chioca et al., 2013), esta falta de acceso también podría expli- car en parte la peor salud oral del sector rural. Otra forma de medir el acceso es por las necesidades de tratamiento no cubiertas, en este caso se mostraron diferencias estadísticamente significativas, siendo mayores las necesidades de tratamiento de anomalías dentomaxilares, restauraciones para el sector rural, esto se explicaría porque las coberturas en el sector urbano son mayores, lo que puede incidir en tratamientos mas precoces que eviten el avance de las patologías. Y por otro lado el acceso restringido de los niños del sector rural a tratamientos por los costos de tiempo, de traslado y de acompañamiento para acceder a la infraestructura en salud (Córdova Villalobos et al., 2011).

En relación a la necesidad de tratamiento de anomalías dentomaxilares interceptables, asociadas a presencia de malos hábitos orales como succión digital, uso prolongado de mamadera y mal posición lingual resultaron significativamente más prevalentes en el sector rural para compresión maxilar y mordida abierta. Semejantes resultados informaron Espinoza \& Parra (2011) en un estudio realizado en la población rural atendida en el Servicio de Salud Viña del Mar/Quillota, obtuvieron que un $29,3 \%$ del total presentó alguna anomalía dentomaxilar interceptable. Esta patologías inciden en la calidad de vida y sería de gran utilidad implementar a distancia por medio de teleodontología programas de ortodoncia preventiva e interceptiva sobre todo en los sectores más vulnerables, con el objetivo de disminuir la prevalencia y el tiempo de tratamiento de anomalías dentomaxilares (Mariño et al., 2014; King et al., 2012; Mandall et al., 2005).

Una de las limitaciones que encontramos fueron los costos de traslado y permanencia de los examinadores en el sector rural dificultando el acceso a zonas profundamente rurales y es probable que en los estudios ya publicados se tendío a recoger los datos de los sectores más conectados, dentro de lo rural, por tanto el daño en salud oral podría estar subestimados.

Este estudio concluye aportando evidencia consistente que la salud oral presentó mayor daño en niños de sector rural constituyéndose la localidad en factor de riesgo para caries, daño periodontal, anomalías dentomaxilares, y menor acceso a los servicios de salud. Se sugieren que se debieran realizar programas promocionales preventivos con estrategias eficientes a nivel rural, enfocados especialmente a los más vulnerables, esfuerzo que debiera hacerse involucrando a los establecimientos educacionales, la comunidad, los padres y a la atención primaria. 
CABRERA, C.; ARANCET, M. I.; MARTíNEZ, D.; CUETO, A. \& ESPINOZA, S. Oral health in urban and rural school population. Int. J. Odontostomat., 9(3):341-348, 2015.

ABSTRACT: Oral health is conditioned by individual local factors and also by socioeconomic, cultural, environmental, community, geographical factors where living in rural or urban areas plays an important role. It is an effort of epidemiological surveillance; the first study in this area that aimed to compare oral health in children aged 6-12 years old in rural and urban locations, using as indicators the presence of caries, periodontal health status and presence of dento-maxillary anomalies. Prevalence study in school population of children aged 6-12 years old from urban and rural locations of Valparaiso Region. The study sample was taken from 231 children who had mixed or permanent dentition. The variables measured were: age, gender, location, presence of mucosa, periodontal and dento maxillary anomalies; dmft/DMFT index, oral hygiene index; presence of sealants and need of treatment; educational level of household head and child caregiver and the employment/ unemployment situation of the household head. The database was analyzed using Microsoft Excel and Stata 13.0. Descriptive statistics and bivariate multivariate inferential statistics were performed. The presence of caries, generalized gingivitis and mucosa lesions were significantly higher in rural areas, as well as, the need of treatment of dento maxillary anomalies, restorations and caries. The average dmft and DMFT index were higher in rural than urban, with significant difference. Through linear model variables such as education of the household head and child caregiver the employment/ unemployment situation of the household head were not significant when trying to explain the variability of DMFT and dmft index, but living in urban or rural areas was an important factor. Oral Health was worse in children from rural areas and constitutes a risk factor for dental caries, periodontal damage, dento maxillary anomalies and less access to health services.

KEY WORDS: oral health, urban health, rural health, dental caries.

\section{REFERENCIAS BIBLIOGRÁFICAS}

Córdova Villalobos, J. A.; Ortiz Domínguez, M. E.; Rodríguez Suárez, R.; Álvarez del Río, F. \& González Retiz, M. L. Guía para la Formulación y Evaluación Económica de Proyectos de Inversión en Salud. México D. F., Centro Nacional de Excelencia Tecnológica en Salud, Dirección de Evaluaciones Tecnológicas para la Salud, 2011.

Espinoza R., A. \& Parra, N. Prevalencia de anomalías dentomaxilares y malos hábitos orales en pre-escolares de zonas rurales de la población beneficiaria del servicio de salud de Viña del Mar/Quillota. Wilde, Salud Dental para Todos, 2011. Disponible en: http://www.sdpt.net/ PADM.htm

Faleiros Chioca, S.; Urzúa Araya, I.; Rodríguez Martínez, G. \& Cabello lbacache, R. Uso de sellantes de fosas y fisuras para la prevención de caries en población infanto-juvenil: Revisión metodológica de ensayos clínicos. Rev. Clin. Periodoncia Implantol. Rehabil. Oral, 6(1):14-9, 2013.

Geus, J. L.; Luca, C. M. B.; Baldani, M. H. \& Czlusniak, G. D. Caries prevalence and self-perception of oral health condition among children of urban and rural schools of Ponta Grossa, PR, Brazil. Pesq. Bras. Odontoped. Clin. Integr., João Pessoa, 13(1):111-17, 2013.

Instituto Nacional de Estadísticas (INE). Censo 2002. Síntesis de Resultados. Santiago de Chile, 2003. Disponible en: http://www.ine.cl/cd2002/sintesiscensal.pdf

King, G. J.; Spiekerman, C. F.; Greenlee, G. M. \& Huang, G. $J$. Randomized clinical trial of interceptive and comprehensive orthodontics. J. Dent. Res., 91(7 Suppl.):59S-64S, 2012.

Mandall, N. A.; O'Brien, K. D.; Brady, J.; Worthington, H. V. \& Harvey, L. Teledentistry for screening new patient orthodontic referrals. Part 1: A randomised controlled trial. Br. Dent. J., 199(10):659-62, 2005.

Mariño, R.; Villa, A. \& Guerrero, S. Programa de fluoración de la leche en Codegua, Chile: evaluación al tercer año. Rev. Panam. Salud Publica, 6(2):117-21, 1999.

Mariño, R.; Villa, A. \& Weitz, A. Prevención de la caries dental utilizando la leche como vehículo para fluoruros: Las experiencias chilenas. Melbourne, Universidad de Melbourne, 2006.

Mariño, R.; Manton, D.; Marwaha, P.; Hallett, K.; Clarke, K.; Hopcraft, M.; McCullough, M. \& Borda, A. The Implementation of Teledentistry for Pediatric Patients. Global Health 2014: The Third International Conference on Global Health Challenges. IARIA, 2015. pp.14-9.

Maupome, G.; Martínez-Mier, E. A.; Holt, A.; Medina-Solís, C. E.; Mantilla-Rodríguez, A. \& Carlton, B. The association between geographical factors and dental caries in a rural area in Mexico. Cad. Saude Publica, 29(7):1407-14, 2013.

Ministerio de Salud (MINSAL). Diagnóstico de Situación de Salud Bucal. Santiago de Chile, Ministerio de Salud, Gobierno de Chile, 2007. 
CABRERA, C.; ARANCET, M. I.; MARTíNEZ, D.; CUETO, A. \& ESPINOZA, S. Salud oral en población escolar urbana y rural. Int. J. Odontostomat., 9(3):341-348, 2015.

Organización Mundial de la Salud (OMS). Encuestas de Salud Bucodental. Métodos Básicos. 4a ed. Ginebra, Organización Mundial de la Salud (OMS), 1997.

Perinetti, G.; Varvara, G. \& Esposito, B. Prevalence of dental caries in schoolchildren living in rural and urban areas: results from the first region-wide Italian survey. Oral Health Prev. Dent., 4(3):199-207, 2006.

Skinner, A. C.; Slifkin, R. T. \& Mayer, M. L. The effect of rural residence on dental unmet need for children with special health care needs. J. Rural Health, 22(1):36-42, 2006.

Soto, L.; Tapia, R.; Jara, G.; Rodríguez, G. \& Urbina, T. Diagnóstico Nacional de Salud Bucal del Adolescente de 12 años y Evaluación del Grado de Cumplimiento de los Objetivos Sanitarios de Salud Bucal 2000-2010. Santiago de Chile, Facultad de Odontología, Universidad Mayor, 2007.

Zander, A.; Sivaneswaran, S.; Skinner, J.; Byun, R. \& Jalaludin, B. Risk factors for dental caries in small rural and regional Australian communities. Rural Remote Health, 13(3):2492, 2013.
Dirección para Correspondencia:

Danitza martinez bordigoni

Cirujano dentista

Facultad de Odontología

Universidad Andrés Bello

Viña del Mar

CHILE

Email: danitza.mb@gmail.com

Recibido : 06-01-2015

Aceptado: 02-09-2015 